\title{
Análisis de perforantes de la epigástrica inferior profunda con Angio TC 3D, Eco Doppler color y Doppler simple de ultrasonidos en colgajo DIEP: resultados preliminares
}

\section{Analysis of deep inferior epigastric perforating vessels with 3D CT angiography, color Doppler ultrasonography and Doppler in diep flaps: preliminary results}

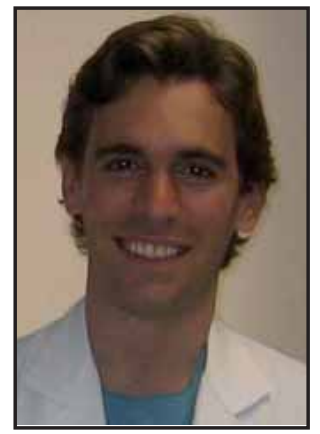

Castro García, J .

\author{
Castro García, J .*, García-Tutor, E.*, Alonso, A.**, Pina, L.**, De Luis, E.**
}

\section{Resumen}

Nuestro objetivo es la validación de la Angio TC tridimensional como herramienta de planificación de los colgajos DIEP, comparándolo con el Doppler de ultrasonidos (US) y eco Doppler color.

Entre enero de 2006 y marzo de 2007 se realiza en 11 pacientes (13 DIEP) un estudio comparativo prospectivo entre el Doppler de US, eco Doppler color y Angio TC con reconstrucción tridimensional, utilizando como dato de referencia los hallazgos intraoperatorios. En dicho proceso se localiza la mejor perforante que pueda servir como pedículo al colgajo DIEP en función de su localización, calibre, trayecto y relaciones anatómicas con respecto al músculo.

La Angio TC con reconstrucción tridimensional, demuestra una especificidad del 100\% (IC 95\% 75.3-100) lo que le convierte en una prueba con un alto valor predictivo positivo y una excelente herramienta en la planificación de los colgajos de perforantes. El eco Doppler color determinó, que tan sólo en un 46,1\% (IC 95\% 19,2-74,9) de los pacientes, la perforante seleccionada por la prueba de forma preoperatoria, coincidía con la perforante elegida en quirófano. Con el Doppler de US, en un 30,8 \% (IC 95\% 9,1-61,4) de los colgajos estudiados, coincidía la mejor perforante escogida de forma preoperatoria, con los hallazgos obtenidos tras la disección del colgajo.

En el presente estudio, la Angio TC tridimensional se ha mostrado como una técnica con una gran especificidad que proporciona valiosa información, sólo comparable con la disección anatómica y por delante de pruebas como el Doppler de ultrasonidos y el eco Doppler color.

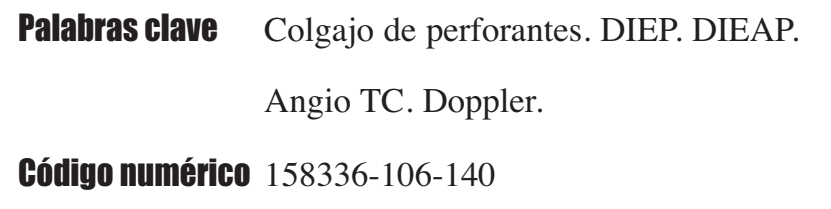

The aim of this report, is to validate the Angio-CT technique with three-dimentional reconstruction as a preoperative planning tool, after comparison with Doppler ultrasound and colorDuplex. Between january 2006 and march 2007, we studied 11 consecutive patients (13 DIEP) in whom a prospective comparative followed up was performed comparing, the findings observed using Doppler ultrasound, Colour-Duplex and Angio-CT with three-dimentional reconstruction. Surgical procedure findings were considered as the reference value. The evaluation procedure included the election of the most suitable perforator vessels, taking account of the location, caliber and anatomical relationships with the muscle.

The Angio-CT with three-dimentional reconstruction showed $100 \%$ specificity (CI 95\% 75.3-100), and very high positive predictive value. In this way, this technique could be considered as an excellent tool for preoperative evaluation of perforators flaps.

Color-Duplex showed that in $46,1 \%$ of the patients (CI $95 \%$ $19,2-74 \%$ ) the selected perforator matched with the intraoperatively selected. Using Doppler ultrasound only in $30,8 \%$ of the cases (IC 95\% 9,1-61,4\%) this result was observed.

In this report, Angio-CT with three-dimentional reconstruction has revealed as a very high specificity technique, that allows a great account of important information, only comparable with anatomical dissection and ahead from Doppler ultrasound and color-Dupplex.

$\begin{array}{ll}\text { Key words } & \begin{array}{l}\text { Perforator flaps. DIEP. DIEAP. CT Angio- } \\ \text { graphy. Doppler sonography. }\end{array} \\ \text { Numeral Code } & 158336-106-140\end{array}$




\section{Introducción}

En la última década los colgajos de perforantes se han popularizado enormemente, ya que presentan la ventaja de respetar la musculatura, disminuyendo enormemente la morbilidad de la zona donante. Aportan la posibilidad de crear colgajos "a medida" basándonos en los territorios vasculares; sin embargo la gran variabilidad anatómica en la distribución del árbol vascular de las perforantes acentúa aún más la exigencia de este tipo de procedimientos en los que un adecuado estudio preoperatorio facilitará en gran medida la correcta ejecución de la intervención.

En nuestro medio hospitalario, el colgajo DIEP (Deep Inferior Epigastric Perforator) es uno de los más utilizados, debido principalmente a su gran utilidad en reconstrucción mamaria. Se trata de un colgajo dermograso abdominal, basado en las perforantes de los vasos epigástricos inferiores (1). Sin embargo, aunque sabemos que las mejores perforantes se localizan alrededor del ombligo, existe una gran variación inter e intrapersonal, que nos impide determinar de forma universal la distribución del plexo vascular que va a determinarlas. Existen varios métodos de localización preoperatoria de las perforantes, aunque quizá los más populares sean el Doppler de ultrasonidos (US), eco Doppler color y últimamente la Angio TC con reconstrucción tridimensional. Hasta la fecha son pocos los artículos en los que se aplican dichas técnicas en la evaluación preoperatoria de los colgajos de perforantes, pero no hemos encontrado ninguno, que compare las tres.

En este estudio trataremos de validar la Angio TC tridimensional como método de evaluación preoperatoria para el colgajo DIEP, comparándolo con el Doppler de US y eco Doppler color y correlacionándolos con los hallazgos intraoperatorios.

\section{CONCEPTOS TEÓRICOS}

Doppler de US: El reflejo de las ondas sonoras, predominantemente del flujo intravascular y del inherente cambio de fase que detecta la sonda según los principios del Doppler, son las bases para la identificación de las arterias y venas del plexo superficial mediante Doppler de US (2). Se trata de una técnica no invasiva que utiliza el medidor de flujo bidireccional Doppler, para valorar el plexo vascular superficial. Es una herramienta compacta, portátil (Fig. 1) y está disponible en la mayoría de los centros debido a su bajo coste $(2,3)$. Es además fácil de utilizar e interpretar, aunque requiere de una curva de aprendizaje para su manejo correcto $(3,4)$.

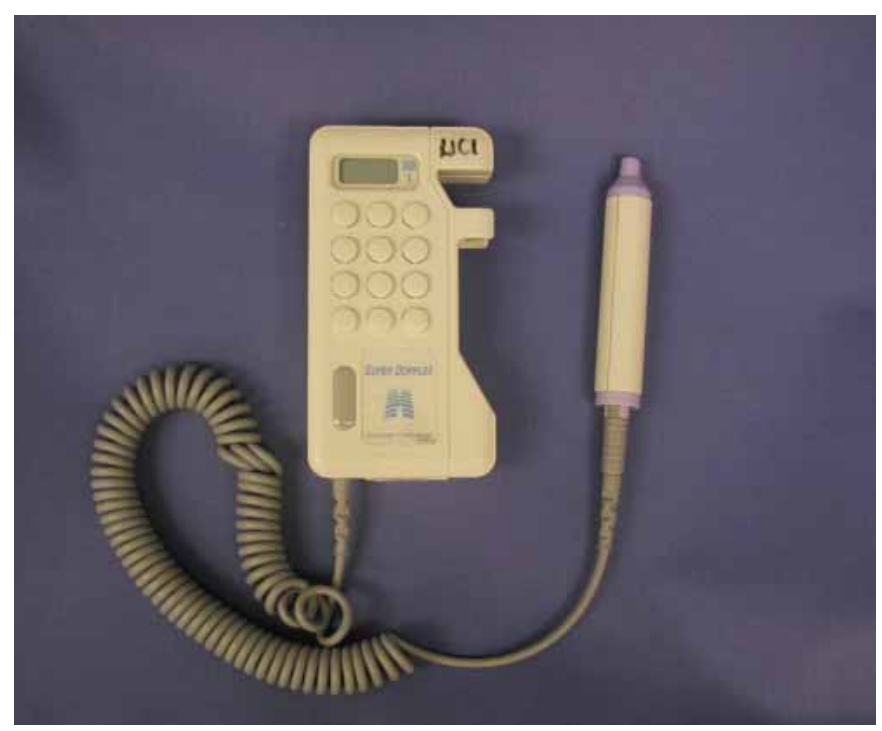

Fig. 1. Doppler de US.

Eco Doppler color: El mismo transductor que se usa para obtener imágenes ecográficas en escala de grises, B-mode, de alta resolución y en tiempo real, puede también detectar un cambio de frecuencia y fase de cualquier objeto que se mueva en el campo que está siendo estudiado. Debido a que las estructuras anatómicas son habitualmente estáticas, esto sólo puede corresponder al flujo sanguíneo (2). Cuando añadimos un espectro de color al componente en movimiento y combinamos ambas técnicas, obtenemos un eco Doppler color. El color que observamos en el monitor, depende de la dirección del flujo sanguíneo en relación con el transductor y arbitrariamente suele ser asignado como azul o rojo. El color rojo indica flujo que se aproxima y el azul flujo que se aleja (Fig. 2).

Angio TC tridimensional: El desarrollo tecnológico que ha experimentado la Tomografía Computarizada (TC) hasta llegar a los actuales TC multicorte de hoy en día, permite el estudio vascular tras la administración de contraste yodado intravenoso, con resultados similares y a veces superiores a la angiografía convencional. Esta técnica, conocida como Angiografía por TC o Angio TC, permite estudiar grandes territorios vasculares en una única adquisición cuyos grosores de corte llegan a ser inferiores al centímetro. A su vez, gracias al análisis tridimensional de la adquisición volumétrica realizada, pueden considerarse imágenes superponibles a la realidad y que no existen en el estudio de una estructura compleja, como puede ser en el árbol vascular de la pared abdominal, y diseñar de una manera no invasiva, exacta y precisa un procedimiento quirúrgico. Esta técnica ha mostrado, en el estudio de los vasos perforantes de la pared abdominal anterior, una excelente correlación entre 

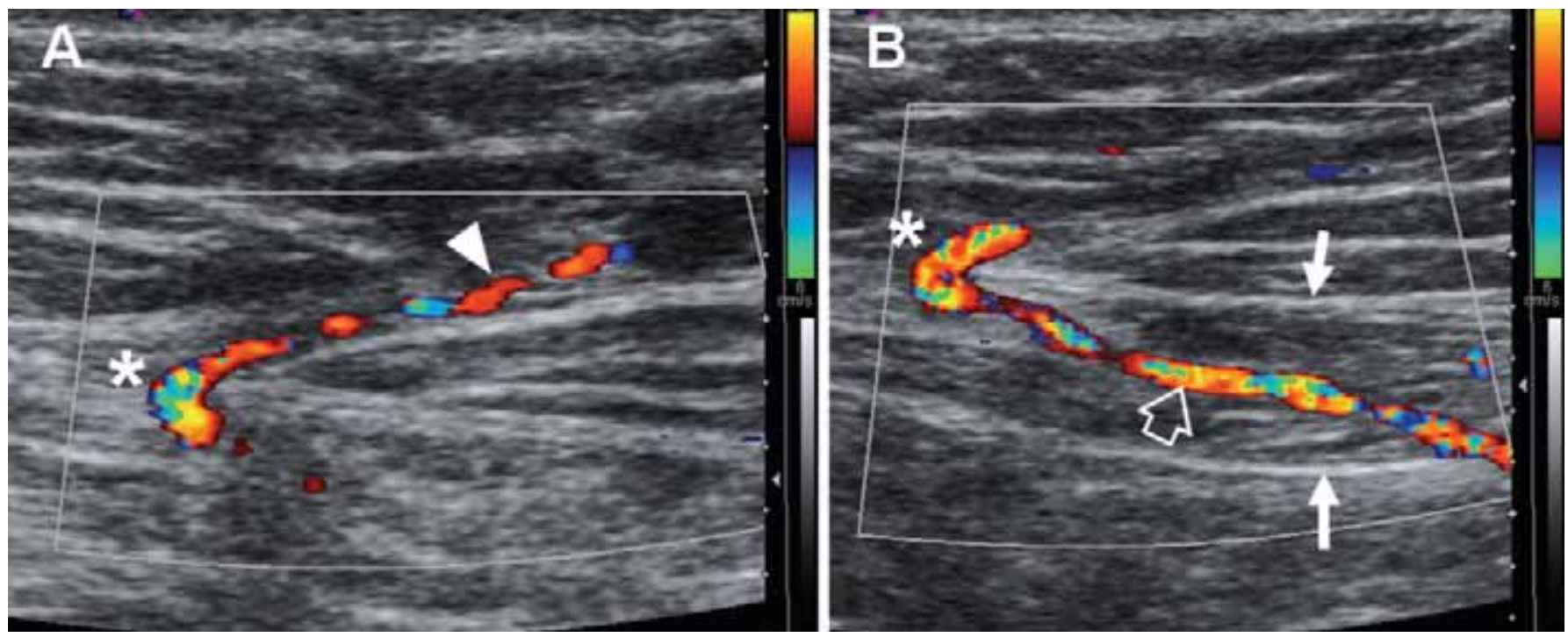

Fig. 2. Imagen de eco Doppler-color en la que se aprecia el músculo recto anterior del abdomen (delimitado por flechas en B) así como el trayecto intramuscular de la perforante (flecha hueca en B). El punto donde atraviesa la fascia superficial del recto anterior $(*$ en A y B) se sigue del trayecto subcutáneo del vaso (cabeza de flecha en A).

los hallazgos obtenidos y los encontrados durante la cirugía, siendo una herramienta de gran utilidad para la planificación de una cirugía de colgajo DIEP $(6,7)$.

\section{Material y método}

Entre enero de 2006 y marzo de 2007 llevamos a cabo un estudio prospectivo en 11 pacientes con edades comprendidas entre 27 y 53 años, en las que se realizaron 13 colgajos DIEP para reconstrucción mamaria: en 9 de ellas unilaterales y en 2 bilaterales (Fig. 3). Obtuvimos el consentimiento informado de las pacientes para su inclusión en el estudio, consistente en la realización de un Doppler de US, eco Doppler color y Angio TC con el fin de valorar preoperatoriamente la localización de la mejor perforante del colgajo.

Doppler de US: utilizamos un Doppler manual bidireccional, modelo SD1 Super Doplex®, (Huntleigh Techonology, Cardiff, U.K) con un transductor de $5 \mathrm{MHz}$, que se aplicó sobre las 11 pacientes a las que se les iban a realizar 13 colgajos DIEP, de forma previa a la realización de eco Doppler color y Angio TC. Todas las exploraciones fueron realizadas por las misma persona (JC), para evitar sesgos. Las pacientes se colocaron siempre en decúbito supino, tal y como iban a estar colocadas en quirófano.

La muestra de 11 mujeres tenía edades comprendidas entre los 27 y los 53 años. Todas ellas iban a ser sometidas a reconstrucción mamaria, en 9 unilateral y en 2 bilateral, mediante colgajo DIEP. Tomando como centro del eje de coordenadas el ombligo, se realizó un rastreo de toda la superficie cutánea abdominal, desde $2 \mathrm{~cm}$ por encima del ombligo, hasta $12 \mathrm{~cm}$ por debajo de éste, extendiendo el rastreo lateralmente 12 cm desde la línea media. El transductor se colocó de forma perpendicular a la superficie cutánea, sobre la que se había aplicado un gel conductor y se rastreó de craneal a caudal y de medial a lateral. Al localizar una señal acústica pulsátil, buscamos el punto de mayor intensidad sonora marcándolo sobre la piel. Posteriormente se selecciono aquella, que por presentar una mayor intensidad sonora, parecía la perforante de mejor calibre. Otro criterio utilizado a la hora de seleccionar los vasos, fue el hecho de que ante condiciones similares, se eligen preferiblemente aquellas que se localizan a nivel periumbilical, ya que en nuestra experiencia son las que van a permitir una mayor viabilidad del colgajo $(8,9)$. Una vez realizado todo el procedimiento, se tomaron fotografías y se transcribieron los hallazgos a unas tablas que serían comparadas posteriormente con los hallazgos obtenidos por el eco Doppler color y la Angio TC, y corroboradas intraoperatoriamente con la disección del colgajo y de las perforantes seleccionadas.

Eco Doppler color: en este estudio se ha utilizado un ecógrafo Siemens Antares (Erlangen Germany) con un transductor de alta frecuencia (> 7,5 MHZ), para obtener imágenes de las perforantes provenientes de la epigástrica inferior profunda que llegan hasta la grasa abdominal. El estudio se realizó sobre las mismas pacientes a las que se les había realizado previamente el Doppler de US y que iban a ser intervenidas para reconstrucción con colgajo DIEP. La prueba fue realizada en todos los casos por un mismo radiólogo (LP), con alta experiencia en ecografía vascular y que desconocía los hallazgos obtenidos mediante Doppler de US y Angio TC para evitar posibles sesgos. Se registraron los hallazgos y se marcaron sobre la piel 

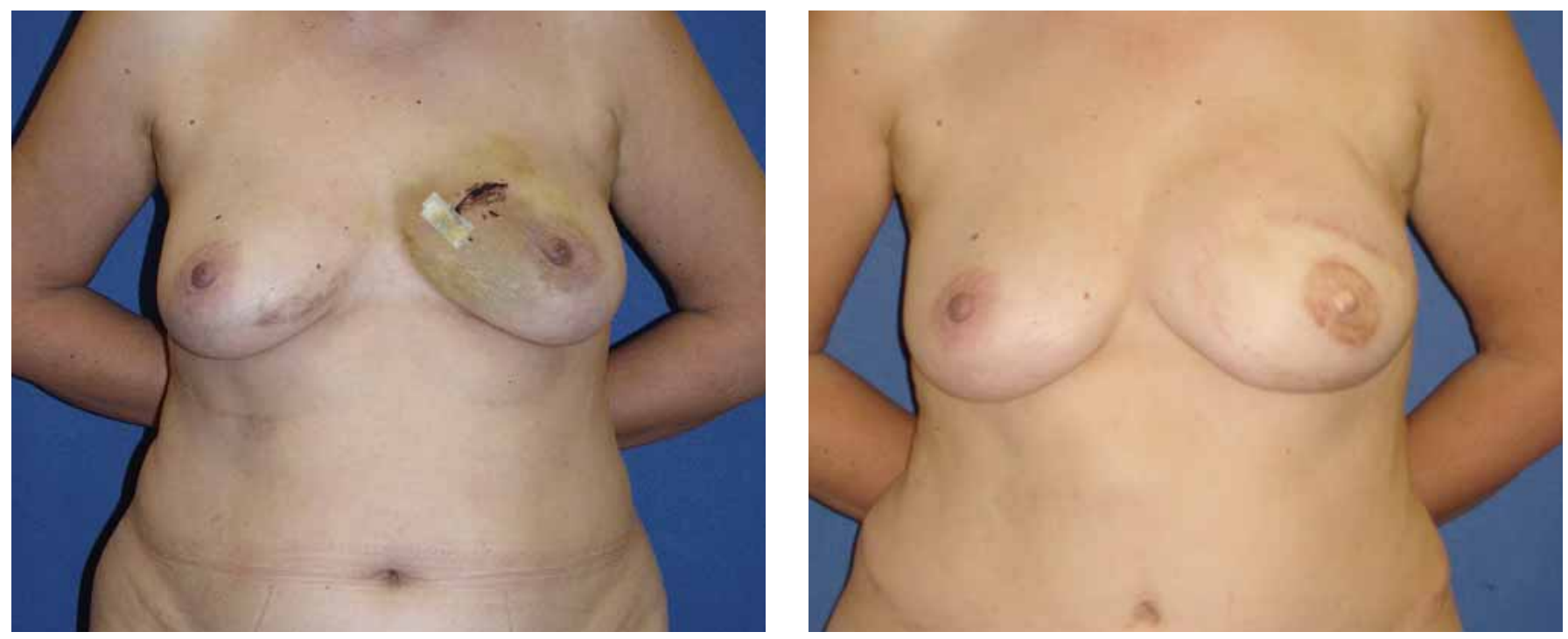

Fig. 3. Imagen pre (A) y postoperatoria (B) de una paciente con reconstrucción mamaria inmediata con DIEP.

las perforantes, seleccionando una como la de elección para basar el colgajo en función de su calibre, localización y recorrido a través del músculo. La exploración duró una media de 45 minutos, tiempo durante el cual la paciente se encontraba en decúbito supino, en la misma posición en la que iba a estar en la mesa de operaciones. Nos aseguramos de que la ropa interior o el pantalón no distorsionaran los hallazgos, evitando cualquier presión a nivel suprapúbico.

El rastreo se realizó tomando como eje de coordenadas el ombligo, al igual que con el Doppler de US y la Angio TC, desde $2 \mathrm{~cm}$. craneales al ombligo hasta $12 \mathrm{~cm}$. caudales al mismo, y a nivel lateral $12 \mathrm{~cm}$. por cada lado, tomando como referencia la línea media del abdomen a nivel umbilical.

Una vez finalizada la prueba, se seleccionó la mejor perforante sobre la que basar el colgajo (entendiendo como perforante al pedículo formado por una arteria, dos venas y en la mayoría de los casos un nervio). Para ello, se eligió la de mayor calibre y con trayecto intramuscular más directo (8-11). Se desecharon aquellas perforantes con trayectos intramusculares largos que harían la disección tediosa, con el riesgo añadido de lesionar los vasos durante la cirugía. Si las características de las perforantes son similares, elegiremos preferiblemente aquellas que se encuentren en la región periumbilical $(6,8)$, que además de mejor calibre, al estar centradas en el colgajo permiten una mayor rotación del mismo una vez realizada la anastomosis con los vasos receptores.

Angio Tc con reconstrucción tridimensional: El estudio preliminar presentado por nuestro grupo de trabajo en el 2006 (6) sobre la realización de Angio TC previa a la cirugía de reconstrucción mamaria mediante colgajo DIEP, mostró un $100 \%$ de concordancia entre los hallazgos obtenidos en la Angio TC y aquellos encontrados durante la cirugía. En dicho trabajo, la mejor perforante descrita en el estudio de angio TC coincidió siempre con aquella escogida en quirófano. Así mismo, se describía con exactitud el trayecto del pedículo vascular, compuesto por una artería y dos venas a través del músculo, así como otros hallazgos anatómicos de interés.

Ante la ausencia de estudios comparativos con otras técnicas de evaluación preoperatoria de vasos perforantes, se inicio un estudio comparativo de Angio TC con Doppler de US y eco Doppler color, cotejando los resultados con los hallazgos intraoperatorios. La realización de Angio TC dura 8 segundos, mientras que la valoración y el postproceso de las imágenes en nuestro estudio duró una media de 20 minutos por paciente.

El estudio de Angio TC se realiza con un período de ayunas previo por la paciente de al menos 6 horas y sin administración de contraste oral. También es necesaria la comprobación de una correcta función renal previa mediante la determinación analítica de creatinina en sangre y la administración previa a la prueba de fluidoterapia oral para una mejor eliminación del contraste y evitar efectos nefrotóxicos. También se obtuvo el consentimiento informado por parte de las pacientes antes de la realización del estudio de Angio TC. En 5 de las pacientes el estudio se realizó en un equipo de TC multicorte de 4 coronas (Somatom Volumen Zoom; Siemens, Erlangen, Germany) y en las restantes en un equipo multicorte de 64 coronas (Somatom Sensation; Siemens, Erlangen, Germany).

Los parámetros técnicos de realización del Angio TC, resumidos en la Tabla I, incluyen la correcta selección del rango de exploración que debe coincidir 

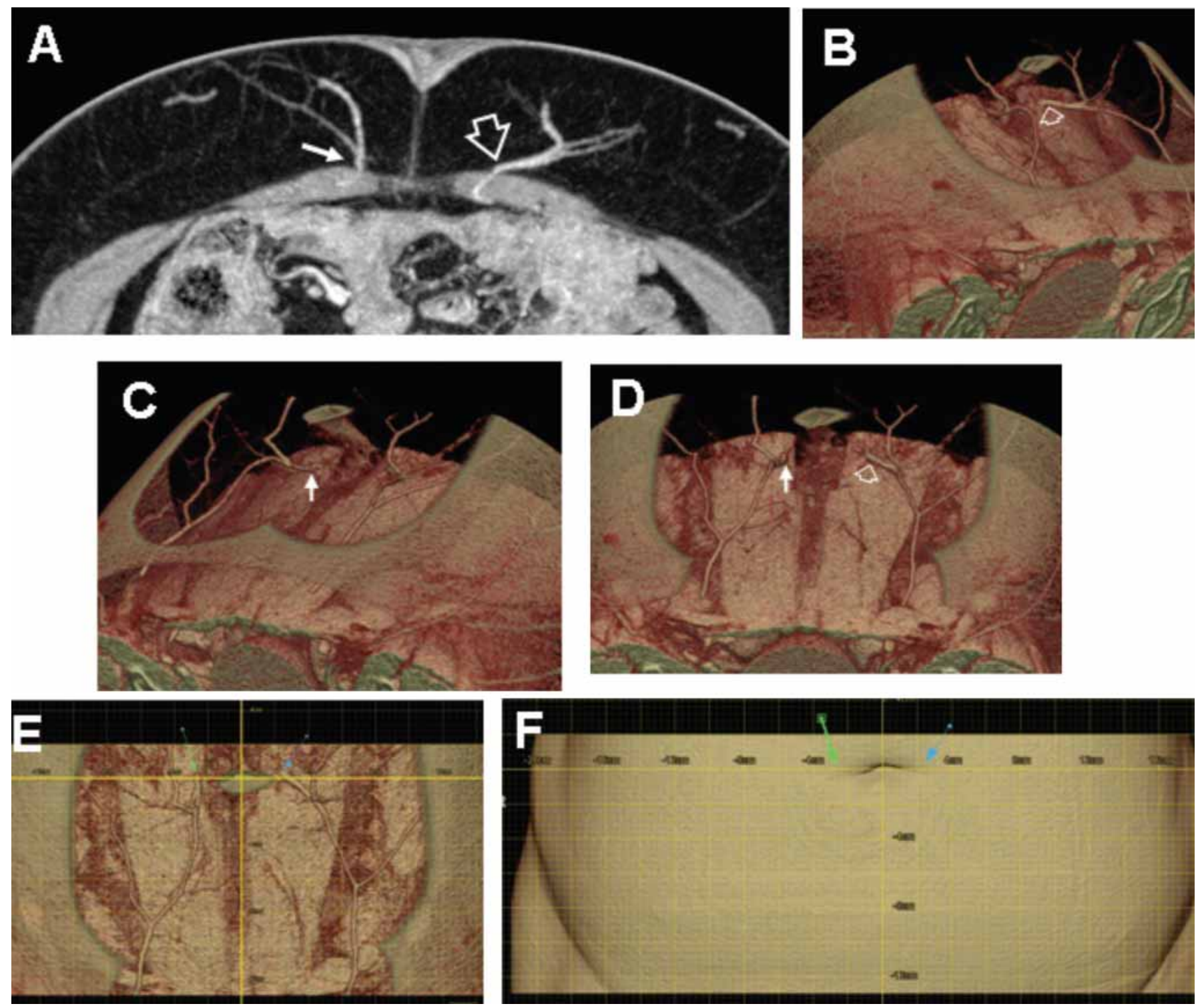

Fig. 4. Los cuatro pasos principales en la localización de la mejor perforante del colgajo DIEP: A) La perforante más gruesa de cada hemiabdomen se localiza en la reconstrucción MIP. B, C, D) En la reconstrucción en 3D VR se marcan las perforantes. E) Con una gradilla milimetrada sobreimpuesta en la imagen y centrada en el ombligo se localizan las coordenadas en una imagen AP. F) Todos estos parámetros son también señalados en la reconstrucción 3D VR.

con aquel propuesto por el cirujano; en este caso incluye un límite por encima del ombligo a $2 \mathrm{~cm}$. y en sentido caudal a $12 \mathrm{~cm}$. Este rango se delimita en el topograma mediante marcadores metálicos que se retiran posteriormente. La adquisición se realiza tras la administración de $150 \mathrm{ml}$ de contraste yodado (270 $\mathrm{mg}$ de yodo por ml, (Visipaque 270; Amersham Health, Little Chalfont, England) con un flujo de $4 \mathrm{ml} / \mathrm{s}$. y técnica de bolus tracking. Las imágenes axiales obtenidas se procesaron por un mismo radiólogo (AA) de manera multiplanar (multiplanar reconstruction MPR), en proyecciones de máxima intensidad (maximun intense proyection MIP) y en reconstrucciones volumétricas (volume rendering VR) cursando con su programa comercial (Inspace, Leonardo, Siemens). Este análisis se realizó de manera ciega y sin conocer el resultado de las otras exploraciones preoperatorias.
Mediante las reconstrucciones MIP, se eligen los vasos perforantes con mejores características siguiendo los mismos criterios de inserción que se utilizaron en el eco Doppler color: mayor calibre, menor trayecto intramuscular y localización preferentemente periumbilical. Una vez localizadas y seleccionadas, en las reconstrucciones VR se establecen en la localización exacta del punto donde estos vasos atraviesan la fascia muscular, punto que a su vez se representa sobre la reconstrucción tridimensional de la piel de la paciente en referencia a un sistema de coordenadas cartesianas cuyo centro se establece en el ombligo (Fig. 4).

Todos los colgajos fueron disecados por el mismo cirujano (EGT). De manera intraoperatoria se eligió la mejor perforante en función de la localización y tamaño, registrándose en su posición y las distintas características para verificar su coincidencia con las distintas técnicas. 
Tabla I. Protocolo de realización de Angio TC de 4 y 64 coronas.

\begin{tabular}{|c|c|c|}
\hline DETECTORES & $4 \times 1$ & $64 \times 0.6$ \\
\hline Topograma & \multicolumn{2}{|c|}{ Con clips metálicos de marcaje del rango } \\
\hline Rango & \multicolumn{2}{|c|}{$2 \mathrm{~cm}$ superior a ombligo hasta $12 \mathrm{~cm}$ por debajo } \\
\hline Bolus Tracking & \multicolumn{2}{|c|}{ Aorta Abdominal lo más próximo a bifurcación iliaca } \\
\hline \multicolumn{3}{|l|}{ Características del Rango } \\
\hline Colimación & $1 \mathrm{~mm}$ & $0.6 \mathrm{~mm}$ \\
\hline $\mathrm{Kv}$ & 120 & 120 \\
\hline mAs & 200 & 200 \\
\hline Tiempo de Rotación & $0.5 \mathrm{~s}$ & $0.5 \mathrm{~s}$ \\
\hline Retraso & $30 \mathrm{~s}$ & $80 \mathrm{~s}$ \\
\hline Pitch & & 0.7 \\
\hline \multicolumn{3}{|l|}{ Contraste } \\
\hline Volumen & $150 \mathrm{cc} / \mathrm{s}$ & $150 \mathrm{cc} / \mathrm{s}$ \\
\hline Velocidad & $4 \mathrm{cc} / \mathrm{s}$ & $4 \mathrm{cc} / \mathrm{s}$ \\
\hline \multicolumn{3}{|l|}{ Flush de suero salino } \\
\hline Volumen & $50 \mathrm{cc}$ & $50 \mathrm{cc}$ \\
\hline Velocidad & $4 \mathrm{cc} / \mathrm{s}$ & $4 \mathrm{cc} / \mathrm{s}$ \\
\hline \multicolumn{3}{|l|}{ Reconstrucción } \\
\hline Slice Thickness & $1.25 \mathrm{~mm}$ & $0.6 \mathrm{~mm}$ \\
\hline Incremento reconstrucción & $0.7 \mathrm{~mm}$ & $0.3 \mathrm{~mm}$ \\
\hline Kernel & $\mathrm{B} 20 \mathrm{f}$ & $\mathrm{B} 10 \mathrm{f}$ \\
\hline
\end{tabular}

Se comparó si la perforante designada como "de elección" con cada una de las técnicas coincidía o no con la elegida intraoperatoriamente; para ello utilizamos el método exacto binomial con el programa Epiinfo versión 6 .

\section{Resultados}

Tras analizar 13 colgajos DIEP realizados en 11 pacientes, obtuvimos que en el $100 \%$ de las pacientes a las que se les realizó la valoración preoperatoria con
Angio TC, el valor predictivo positivo (VPP) para hallar la mejor perforante, comparándolo con los hallazgos intraoperatorios (Tabla II), fue del $100 \%$ (IC 95\% 75.3-100) hallado por el método exacto binomial con el programa Epi-info versión 6 .

Sin embargo, los resultados obtenidos tras realizar la prueba de eco Doppler color determinó que tan sólo en un 46,1\% (IC 95\% 19,2-74,9) de los pacientes la perforante seleccionada por la prueba de forma preoperatoria coincidía con la perforante elegida en quirófano.

Tabla II: Resumen de los hallazgos obtenidos con las tres pruebas.

Doppler de US: 4/13: coincide la perforante seleccionada por la prueba, con la mejor perforante del quirófano. Eco Doppler color: 6/13: coincide la perforante seleccionada por la prueba, con la mejor perforante del quirófano. Angio TC: 13/13: coincide con la mejor perforante del quirófano.

\begin{tabular}{lccc} 
Coppler US & Eco Doppler color & Angio TC \\
Colgajo & Coincide con los hallazgos quirúrgicos & Coincide con los hallazgos quirúrgicos & Coincide con los hallazgos quirúrgicos \\
1 & $\mathrm{No}$ & $\mathrm{No}$ & $\mathrm{Si}$ \\
2 & $\mathrm{Si}$ & $\mathrm{Si}$ & $\mathrm{Si}$ \\
3 & $\mathrm{Si}$ & $\mathrm{Si}$ & $\mathrm{Si}$ \\
4 & $\mathrm{Si}$ & $\mathrm{No}$ & $\mathrm{Si}$ \\
5 & $\mathrm{No}$ & $\mathrm{No}$ & $\mathrm{Si}$ \\
6 & $\mathrm{No}$ & $\mathrm{No}$ & $\mathrm{Si}$ \\
7 & $\mathrm{No}$ & $\mathrm{No}$ & $\mathrm{Si}$ \\
8 & $\mathrm{No}$ & $\mathrm{No}$ & $\mathrm{Si}$ \\
9 & $\mathrm{No}$ & $\mathrm{Si}$ & $\mathrm{Si}$ \\
10 & $\mathrm{No}$ & $\mathrm{Si}$ & $\mathrm{Si}$ \\
11 & $\mathrm{Si}$ & $\mathrm{Si}$ & $\mathrm{Si}$ \\
12 & $\mathrm{No}$ & $\mathrm{No}$ & $\mathrm{Si}$ \\
13 & $\mathrm{Si}$ & $6 / 13$ & $\mathrm{Si}$ \\
\hline
\end{tabular}


En cuanto a los resultados obtenidos con el Doppler de US, en un 30,8\% (IC 95\% 9,1-61,4) de los colgajos estudiados, coincidía la mejor perforante escogida de forma preoperatoria, con los hallazgos obtenidos tras la disección del colgajo.

La Angio TC no es capaz de determinar con exactitud el calibre del pedículo vasculonervioso aunque, con la reconstrucción tridimensional, nos permite compararar las distintas perforantes y de esta forma elegir las de mayor calibre.

\section{Discusión}

El reflejo de las ondas sonoras del flujo intravascular que detecta el transductor Doppler de US es la base para la localización de las arterias y venas del plexo superficial $(2,3)$.Se trata de una técnica no invasiva que utiliza el medidor de flujo bidireccional Doppler para valorar la localización de los vasos perforantes sobre los que se va a basar nuestro colgajo. Es una herramienta compacta, portátil y está disponible en la mayoría de los centros debido a su bajo coste. Presenta la ventaja de ser fácil de utilizar e interpretar aunque, como ya refería Taylor en sus estudios anatómicos sobre los angiosomas, requiere de una curva de aprendizaje: "al igual que el estudiante de Medicina que debe familiarizarse con los complicados ruidos y murmullos cardiacos en la facultad" $(1,2)$. Pequeñas variaciones en la presión de la sonda sobre la piel pueden alterar la percepción. Una presión excesiva puede hacer que los vasos de pequeño calibre se colapsen y sean por tanto imperceptibles, o que por el contrario, estemos detectando vasos que se encuentran en la profundidad y que no llegan a alcanzar la grasa subdérmica. De la misma forma, una presión insuficiente sobre la piel puede hacer que no lleguemos a detectar los vasos que buscamos. Requiere por tanto, de una curva de aprendizaje para aplicar la presión adecuada sobre la superficie cutánea del área a estudiar y para familiarizarse, identificar y relacionar los diferentes sonidos que nos encontramos, con la calidad de las perforantes que buscamos.

En ocasiones, la correcta salida de las perforantes no puede ser detectada, sobre todo cuando su origen está en grandes vasos, debido al ruido de fondo existente. En ocasiones, cuando los vasos axiales son superficiales, podemos llegar a confundirlos con perforantes dando lugar a falsos positivos (8). Otras veces, un panículo dermograso importante puede distorsionar los hallazgos. Tenemos que tener en cuenta también que la percepción es subjetiva, e incluso cuando la prueba la realiza la misma persona, puede llevarnos a errores. Es por tanto una herramienta que puede sernos de gran utilidad en el seguimiento postoperatorio en Microcirugía, al detectar fácilmente la permeabilidad de la anastomosis. Su portabilidad, lo hace muy útil en ciertas ocasiones, como es su uso intraoperatorio; sin embargo, la gran sensibilidad que presenta, unida a la baja especificidad que hemos encontrado, limita su utilidad en la valoración preoperatoria de las perforantes en el colgajo DIEP. No obstante, puede sernos de utilidad para la valoración de las perforantes en aquellos colgajos que presentan una anatomía vascular constante.

El eco Doppler color se ha usado desde hace muchos años para evaluar grandes vasos. En el campo de la Cirugía Plástica Reparadora, cirujanos como Hallock o Blondeel fueron grandes precursores de esta técnica, usándola ampliamente en la evaluación preoperatoria de los vasos perforantes musculocutáneos y fasciocutáneos (2,3,8-11). El eco Doppler color es una técnica no invasiva, presente en la mayoría de los hospitales, que tiene la gran ventaja de no aplicar radiación al paciente. Requiere un personal especializado para su realización y es muy importante que el cirujano esté presente junto al radiólogo para clarificar los objetivos del estudio y explicar, exactamente, lo que quiere valorar. Es fundamental que el radiólogo conozca perfectamente la anatomía vascular de la región de la que vamos a obtener el colgajo y así optimizar la búsqueda.

El eco Doppler color nos permite conocer con mayor exactitud que el Doppler de US la calidad de los vasos que vamos a encontrarnos durante la disección; así mismo permite conocer el trayecto de los vasos a través de las distintas estructuras. Sin embargo, las imágenes ecográficas son muchas veces difíciles de interpretar por los cirujanos, lo que puede llegar a ser una fuente de errores. Por otro lado, muchas veces es difícil discernir si los vasos atraviesan la fascia muscular y penetran correctamente en la grasa, pudiendo dar lugar a falsos negativos. El eco Doppler color no es capaz de determinar el tamaño de las perforantes, aunque sí nos da una idea de cuál de las presentes tiene un calibre mayor. Otro inconveniente es la duración de la prueba que habitualmente suele oscilar alrededor de los 45 minutos. Es por tanto una buena prueba para la realización de una valoración preoperatoria de las perforantes en el colgajo DIEP; sin embargo, la exigencia de un personal altamente cualificado para su realización y la dificultad que para la mayoría de los cirujanos plásticos supone la interpretación de este tipo de imágenes, así como la dificultad que supone el transportar las imágenes al quirófano, hace que no alcance los niveles de éxito de la Angio TC con reconstrucción tridimensional.

La Angio TC, que en un principio estuvo reservada a la valoración de patología inflamatoria y tumoral, poco a poco fue desarrollándose hasta llegar al estudio 
de grandes vasos y posteriormente a vasos de pequeño calibre. Esto nos ofreció la posibilidad de valorar preoperatoriamente los territorios vasculares de distintas áreas del cuerpo, lo que unido a las posibilidades que nos ofrece la reconstrucción tridimensional, hace que esta prueba no sólo nos permita conocer con exactitud las características de vasos de pequeño calibre, sino también el trayecto de los mismos y su relación con las estructuras adyacentes. También ofrece la posibilidad de conocer datos anatómicos de interés a la hora de planificar una intervención quirúrgica al explorar en realidad virtual el campo quirúrgico que vamos a encontrarnos. Además, evitamos los falsos positivos y negativos operador-dependientes que se presentan con el Doppler de US o el eco Doppler color al tratarse de una técnica que no es tan dependiente del radiólogo como las anteriores. La reconstrucción tridimensional de la Angio TC nos aporta una visión global de todo el campo operatorio, de forma que de un solo vistazo podemos ver todas las perforantes que nos pueden ser útiles, las posibles anastomosis con el sistema superficial y la situación de éste en el colgajo. Es obvio que aporta más información una imagen en tres dimensiones que un corte en sólo dos. Los cortes transversales de la angio TC nos dan información precisa sobre el trayecto intramuscular de los vasos epigástricos inferiores profundos, pero no de la arborización de la perforante en el espesor de la grasa, ni las anastomosis entre unas y otras perforantes o con las contralaterales. Todos estos datos son los que nos permiten elegir preoperatoriamente la mejor perforante del colgajo, no sólo por ser la de mayor calibre, sino también por presentar mejor situación en el colgajo, ser la más fácil de disecar y la que presenta mayor número de anastomosis con el resto de perforantes y el sistema superficial. Gracias a la Angio TC con reconstrucción tridimensional, cuando el cirujano está disecando el colgajo tiene la sensación de haber estado allí antes, de forma que la cirugía es más rápida y segura, permitiendo tener un plan alternativo en caso de lesión accidental de los vasos durante la disección.

Las limitaciones de la Angio TC son la radiación que se aplica a los pacientes, que viene a ser equivalente a la aplicada en una radiografía completa de columna o en un enema opaco, y la necesidad de aplicar contraste yodado, por lo que debemos comprobar que los pacientes presentan una adecuada función renal antes de someterlos a la prueba. Otro factor limitante es el costo de la prueba misma, que varía de unos países a otros y que en nuestro medio viene a ser el doble que el de un eco Doppler color, si bien, debemos considerar el tiempo quirúrgico que vamos a ahorrar al permitirnos disecar directamente la perforante seleccionada, así como la seguridad que nos ofrece la prueba al permitirnos conocer con exactitud las características del vaso que pretendemos disecar. Otra limitación a tener en cuenta son las alergias al contraste yodado que lógicamente nos obligaría a hacer uso del eco Doppler color para elegir la mejor perforante. Por ahora no hemos tenido que omitir ninguna Angio TC preoperatoria por este motivo; solo una paciente presento prurito en zona perineal secundariamente al uso del contraste que se resolvió espontáneamente y sin complicaciones.

A la vista de los resultados obtenidos en este estudio, la Angio TC con reconstrucción tridimensional, es la prueba que ofrece mayores garantías, obteniendo un valor predictivo positivo del $100 \%$ en la localización de la perforante dominante. Además, nos ofrece la posibilidad de conocer datos anatómicos de interés, como la presencia o no de un sistema epigástrico superficial adecuado, el trayecto de los vasos, la existencia de variantes anatómicas vasculares o el estado de la musculatura abdominal.

El futuro en este campo de la Cirugía Reparadora pasa por encontrar un método de diagnóstico no invasivo, que sea portátil, sencillo, barato y seguro para los pacientes. Hasta ese momento, la Angio TC con reconstrucción tridimensional quizá sea nuestro mejor aliado en la valoración preoperatoria de las perforantes del colgajo DIEP.

\section{Conclusiones}

La Angio TC con reconstrucción tridimensional está por delante de pruebas tradicionalmente utilizadas en la valoración preoperatoria de los colgajos de perforantes como son el Doppler de US y el eco Doppler color, que si bien pueden ser muy útiles en determinadas circunstancias, no alcanzan el valor predictivo positivo de la Angio TC, solamente comparable con la disección anatómica.

\section{Agradecimientos}

Agradecemos al Dr. Guillén del Departamento de Medicina Preventiva de la Clínica Universitaria su ayuda en el estudio estadístico. También a Eva Morillo y Cristina Guillén por su inestimable ayuda coordinando las distintas exploraciones.

\section{Dirección del autor}

Dr. Javier Castro García

Servicio de Cirugía Plástica

Hospital Universitario La Paz

Madrid. España.

e-mail: j.castro.garcia@hotmail.com 


\section{Bibliografía}

1. Koshima I., Soeda S.: "Inferior epigastric artery skin flaps without rectus abdomininis muscle”. Br J Plast Surg 1989; 42 (6): 645.

2. Hallock G.G.: "Doppler sonography and color duplex imaging for planning a perforator flap". Clin Plastic Surg 2003; 30 (3): 347.

3. Hallock G.G.: "Evaluation of Fasciocutaneous Perforators Using Color Duplex Imaging". Plast Reconstr Surg 1994; 94(5): 644.

4. Taylor G.I., Doyle M., y MC Carten G.: "The Doppler probe for planning flaps: Anatomical study and clinical applications". Br J Plast Surg 1990; 43(1): 1.

5. Taylor G.I, Palmer J.H.: "The vascular terrritories (angiosomes) of the body: experimental study and clinical applications". Br J Plast Surg 1987; 40 (2):113.

6. Alonso-Burgos A., Garcia-Tutor E., Bastarrika G., Cano D., Martínez-Cuesta A., Pina L.J.: "Preoperative planning of deep inferior epigastric artery perforator flap reconstruction with multis-
lice-CT angiography: imagin findings and initial experience". J Plast Reconstr Aesthet Surg 2006; 59 (6): 585.

7. Saber A.A., Meslemani A.M., Davis R., Pimentel R.: "Safety Zones for Anterior Abdominal Wall Entry During Laparoscopy. A CT Scan Mapping of Epigastric Vessels". Ann Surg 2004; 239 (2): 182.

8. Blondeel P.N., Beyens G., Verhaeghe R., Van Landuyt P., Tonnard P., Monstrey S.J., Matton G.: "Doppler flowmetry in the planning of perforator flaps". Br J Plast Surg 1998; 51 (3): 202.

9. Giunta R.E., Geisweid A., Feller A.M.: "The value of preoperative Doppler sonography for planning free perforator flaps". Plast Reconstr Surg 2000; 105 (7): 2381.

10. Masià J., Clavero JA., Carrera A.: "Planificación preoperatoria de los colgajos de perforantes". Cir. plást. iberolatioam. 2006; 32 (4): 237.

11. Blondeel P.N., Morris S.F., Hallock G.G., Neligan P.C.: "Perforators flaps, Anatomy, Technique \& Clinical Aplications". St Louis: Quality Medical Publishing; 2006. 


\title{
Comentario al trabajo uAnálisis de perforantes de la epigástrica inferior profunda con Angio TC 3D, Eco Doppler Color y Doppler simple de ultrasonidos en colgajo DIEP: resultados preliminaresı
}

\author{
Dres. Alexandre Mendonça Munhoz y Marcus Castro Ferreira \\ Departamento de Cirugia Plástica. Universidad de São Paulo. São Paulo. Brazil.
}

Hemos leído con interés el artículo presentado por Castro Garcia et al. Coincidimos plenamente en que se trata de una ingeniosa y probablemente eficaz nueva estrategia de evaluación preoperatoria para el colgajo DIEP. Nos complace constatar el hecho de que la cirugía de reconstrucción mamaria está evolucionando hacia procedimientos que nos permiten realizar reconstrucciones de mayor calidad y durabilidad de resultados en el tiempo, con una menor morbilidad para la paciente. Los colgajos de perforantes se desarrollan como recurso para solventar dicho problema, ya que apenas dañan el músculo y su fascia. El colgajo DIEP ha demostrado ser una alternativa con bajas tasas de complicaciones locales, debido a la falta de sacrificio del músculo recto abdominal, e importante grado de satisfacción (1-6). Como inconveniente presenta su mayor dificultad técnica y la necesidad de un equipo quirúrgico que domine la microcirugía vascular.

La radiología ha experimentado un progreso técnico espectacular en la última década. Los años noventa han sido testigos del desarrollo de la TC helicoidal, con las múltiples posibilidades de reconstrucción bidimensional y tridimensional y de obtención de estudios angiográficos que, en muchos casos, suplantan a la angiografía convencional. En esta década también han explotado las posibilidades de la RM, como las secuencias de difusión, perfusión, espectroscopía y angiografía. Estas técnicas, que no siempre están disponibles, abren nuevas posibilidades en el diagnóstico de la perforante dominante y pueden ser muy útiles en el estudio de la anatomía del abdómen. El desarrollo de la Angio-TC, con imágenes del sistema vascular en tres dimensiones, permite tener un conocimiento exacto de la anatomía de la paciente antes de la intervención, cuestión fundamental para el éxito de la reconstrucción. El procedimiento quirúrgico cobra así mayor rapidez y seguridad al conocer el facultativo de antemano las características de la vascularización. Cuando la evaluación ecocardiográfica no permite una definición anatómica, la Angio TC puede ofrecer detalles que en determinados casos reemplazan la angiografía convencional.

Creemos que en la reconstrucción mamaria, el tiempo quirúrgico prolongado y lo blando de la lesión del pedículo perforante en la disección intramuscular son los principales cuestionamentos al beneficio del DIEP frente al colgajo TRAM convencional. Estos dos fatores pueden ser consecuencia de la técnica y de los detalles anatómicos de los vasos perforantes en su recorrido por el músculo. La posibilidad de prever anatomicamente la localización y el tipo de trayecto intramuscular, favorecen la disección del colgajo. El estudio preliminar presentado por nuestro grupo en el 2004 con base en estudios anatómicos en cadáveres y la experiencia clínica proponen la planeación intraoperatoria en la elección de los vasos perforantes (2). De acuerdo con el protocolo de investigación, los vasos perforantes tienen dos patrones distintos de trayecto intramuscular según la posición y relación con los ramos de la arteria epigastrica profunda inferior. Existe un trayecto, denominado rectilíneo, cuyo recorrido intramuscular es menor y se localiza en un solo septo intermuscular. En otro trayecto, denominado oblicuo, el recorrido es mayor y no se localiza en un solo septo intermuscular. En los vasos de la región lateral del músculo recto se observó un trayecto rectilíneo en $79 \%$ de los vasos disecados, mientras que de los vasos localizados en la región medial solo $18 \%$ presentan esta distribución. La dificultad técnica de la disección de la artéria perforante en el trayecto intramuscular oblicuo es el gran inconveniente para que se difunda ampliamente la técnica. Además, el nivel de dificultad de la disección puede estar relacionado con el riesgo mayor de lesión iatrogénica del vaso perforante y con tiempo operatorio más prolongado. Con las imágenes tridimensionales, el cirujano obtiene una visión global, no solo de la anatomía vascular, sino de todo el área quirúrgica, en este caso de la pared anterior del abdomen, pudiendo observarla desde todos los puntos de vista.

Pensamos que existe una controversia sobre el área receptora del colgajo. Sin embargo, hay diversos aspectos que se deben considerar. A nuestro juicio entre las características importantes en la elección del pedículo receptor en la región torácica se pueden destacar la compatibilidad de calibre, la anatomia constante y la proximidad a la región de reconstrucción. Nos parece más ventajosa la realización de la anastomosis en los vasos mamarios internos, ya que no altera la región axilar y evita así el agravamiento del linfedema que suele acompañar a la combinación disección axilar-radioterapia. Los vasos mamários internos están más cerca de la reconstrucción y no presentan los inconvenientes de la manipulación quirúrgica previa y la radioterapia, características de las reconstrucciones tardias (6). Además, los ramos perforantes del músculo pectoral son una buena alternativa de pedículo receptor, ya que su localización es semejante a la de los vasos mamarios internos y no comprometen la cirugía cardíaca en el futuro. El estudo clínico presentado por nuestro grupo (3), revela que en el $70 \%$ se comprobó la presencia del vaso perforante del segundo espacio intercostal y la anastomosis con el vaso perforante fue posible en el $42 \%$ de los casos. La incompatibilidad de calibre entre vasos receptores y pedículo del colgajo llevan al cirujano a evaluar otras alternativas o a contraindicar el procedimiento. En estas situaciones, es preciso realizar previamente el examen Angio TC de la región en cuestión y garantizar el flujo vascular en estos pedículos antes de programar la reconstrucción.

En conclusión, el desarrollo de la Microcirugía ha permitido mejorar la calidad de las reconstrucciones: gracias a esta técnica es posible reconstruir prácticamente toda clase de defectos, reduciendo la morbilidad para el paciente. La reconstrucción con los colgajos perforantes constituye un claro ejemplo de las ventajas de las técnicas microquirúrgicas. Con el colgajo DIEP el cirujano se acerca a la reconstrucción ideal, proporcionando un buen resultado estético, completamente autógeno, perdurable en el tiempo, con una mínima morbilidad asociada al procedimiento, lo que ayuda en definitiva a recuperar rápidamente el defecto físico y la autoestima perdida. No obstante, somos conscientes de que la reconstrucción con colgajos microquirúrgicos tampoco está exenta de problemas, fundamentalmente relacionados con la técnica, ya que lleva implícita un exquisito manejo y los resultados se rigen por la norma del todo o nada (si falla la técnica la necrosis del colgajo es total).

La incorporación de la Angio TC para la obtención de imágenes angiográficas permite la reconstrucción tridimensional o en cualquier plano de los vasos del sector explorado. Las imágenes vasculares son directas y no están influidas por el flujo, turbulencia o dirección de los vasos, siendo de fácil interpretación. Presenta el inconveniente de requerir la administración de contraste radiopaco para cada adquisición de imágenes. Además, en pacientes poco colaboradores, debido a los movimientos respiratorios, pueden aparecer interferencias que disminuyan la calidad de la exploración. La radiación a la que se somete el paciente es escasa, debido a la rapidez de la realización del estudio. Además, al indicar una Angiotomografía se debe conocer con exactitud lo que se pretende estudiar, lo que minimiza la exposición a la radiación en el paciente. En la actualidad se considera que, aún siendo una técnica muy válida, se precisan estudios comparativos que permitan determinar su fiabilidad.

En cualquier caso, y pese a las consideraciones realizadas que no tratan más que de aproximar la práctica clínica a la excelencia, así como de hacernos reflexionar sobre aspectos concretos que por conocidos no debieran ser descuidados, no quisiéramos obviar lo 
acertado del concepto del análisis de perforantes de la epigástrica inferior profunda com angio TC 3D presentado por los autores, estrategia que seguro merece ser ulteriormente explorada.

Bibliografía

1. Munhoz AM, Ishida LH, Duarte GG, Cunha MS, Montag E, Sturtz G, Gemperli R, Ferreira MC.: "Aesthetic refinements in breast augmentation with deep inferior epigastric perforator flap". Aesth. Plast Surg. 2003; 27: 107.

2. Munhoz AM, Ishida LH, Sturtz GP, Cunha MS, Montag E, Saito FL, Gemperli R, Ferreira MC.: "Importance of lateral row perforator vessels in deep inferior epigastric perforator flap harvesting". Plast Reconstr Surg. 2004; 113: 517.

3. Munhoz AM, Ishida LH, Montag E, Sturtz GP, Saito FL, Rodrigues L, Gemperli R, Ferreira MC.: "Perforator flap breast recons- truction using internal mammary perforator branches as a recipient site: an anatomical and clinical analysis". Plast Reconstr Surg. 2004; 114: 62 .

4. Munhoz AM, Sturtz GP, Montag E, Arruda EG, Aldrighi C, Gemperli R, Ferreira MC.: "Clinical outcome of abdominal wall after deep inferior epigastric perforator (DIEP) flap harvesting and immediate application of abdominoplasty techniques". Plast Reconstr Surg. 2005; 116: 1881.

5. Munhoz AM, Arruda E, Montag E, Aldrighi C, Aldrighi JM, Gemperli R, Ferreira MC.: "Immediate skin-sparing mastectomy reconstruction with deep inferior epigastric perforator (DIEP) flap. Technical aspects and outcome". Breast J. 2007; 13(5):470.

6. Munhoz AM, Duarte GG, Fells K, Arruda E, Montag E, Aldrighi C, Aldrighi JM, Ferreira MC.: "The use of a deep inferior epigastric perforator flap (DIEP) in a case of severe breast asymmetry secondary to radiation therapy during childhood". Breast J. 2007; 13(1):76.

\section{Respuesta al comentario de los Dres. Alexandre Mendonça Munhoz y Marcus Gastro Ferreira}

\section{Dr. J avier Castro García}

En primer lugar agradecer los comentarios realizados por los Dres. Alexandre Mendonça Munhoz y Marcus Castro Ferreira que demuestran la gran experiencia que tienen con el colgajo DIEAP.

Efectivamente, como ya se ha destacado en el comentario al trabajo, la reconstrucción de mama ha evolucionado enormemente en los últimos años, gracias en parte al gran desarrollo y creciente implantación que han adquirido las técnicas microquirúrgicas y radiodiagnósticas en los hospitales de nuestro medio en la última década. Todo ello ha favorecido la popularización de los colgajos de perforantes y en especial del DIEAP y el SIEAP, que se han consolidado como dos de las principales opciones a tener en cuenta en la reconstrucción mamaria, tanto inmediata como diferida.
No hay que olvidar que se trata de intervenciones quirúrgicas complejas, en las que existe una gran variabilidad anatómica inter e intrapersonal, que nos impide saber el punto de salida de la perforante de elección. En este sentido la Angio-TC con reconstrucción tridimensional nos es de gran ayuda, ya que nos permite crear un campo quirúrgico virtual que predice con gran exactitud aquello que nos vamos a encontrar en la intervención, ayundándonos a planificar mejor la cirugía, contribuyendo a disminuir el tiempo operatorio y en definitiva, disminuyendo la morbilidad de los colgajos.

Con esto no quiero menospreciar las otras pruebas diagnósticas objeto del estudio, que si bien no presentan la sensibilidad y especificidad de la Angio-TC, tienen también su utilidad en la planificación preoperatoria microquirúrgica. 